\title{
Differences in Utilisation of the General and Paediatric Emergency Departments by Paediatric Patients
}

\author{
Jacqueline CL Tan, ${ }^{1}$ MBBS (S'pore), MMED (Emerg Med), Peck Har Ang, ${ }^{2}$ MBBS (S'pore), MCEM (UK), Shu-Ling Chong, ${ }^{3}$ MRCPCH (UK), MCI, MPH, \\ Khai Pin Lee, ${ }^{3}$ MBBS (S'pore), MRCPCH (UK), MMED (Paeds), Gene YK Ong, ${ }^{3}$ MBBS (S'pore), MRCPCH (UK), \\ Nur Diana Binte Zakaria, ${ }^{4}$ MBBS (S'pore), MMED (Emerg Med), Jen Heng Pek, ${ }^{1}$ MBBS (S'pore), MCEM (S'pore), MMED (Emerg Med)
}

\begin{abstract}
Introduction: Paediatric patients presenting to the general emergency departments (EDs) differ from those presenting to paediatric EDs. General EDs vary in preparedness to manage paediatric patients, which may affect delivery of emergency care with varying clinical outcomes. We aimed to elucidate the differences in utilisation patterns of paediatric and general EDs by paediatric patients.

Methods: This study was conducted in a public healthcare cluster in Singapore consisting of 4 hospitals. A retrospective review of the medical records of paediatric patients, defined as age younger than 16 years old, who attended the EDs from 1 January 2015 to 31 December 2018, was performed. Data were collected using a standardised form and analysed.

Results: Of the 704,582 attendances, 686,546 (97.4\%) were seen at the paediatric ED. General EDs saw greater number of paediatric patients in the emergent (P1) category (921 [5.1\%] versus $14,829[2.2 \%] ; P<0.01)$ and those with trauma-related presentations $(6,669$ [37.0\%] vs $108,822[15.9 \%] ; P<0.01)$. The mortality of paediatric patients was low overall but significantly higher in general EDs $(39[0.2 \%]$ vs $32[0.005 \%] ; P<0.01)$. Seizure, asthma/bronchitis/bronchiolitis, allergic reaction, cardiac arrest and burns were the top 5 diagnoses that accounted for 517 (56.1\%) of all emergent (P1) cases seen at general EDs.

Conclusion: General EDs need to build their capabilities and enhance their preparedness according to the paediatric population they serve so that optimal paediatric emergency care can be delivered, especially for critically ill patients who are most in need of life-saving and timely treatment.
\end{abstract}

Ann Acad Med Singap 2020;49:948-54

Keywords: Attendance, emergency, epidemiology, health services, paediatrics

\section{Introduction}

The practice of paediatric emergency medicine involves the provision of accessible and timely medical care to acutely ill children and their families. Children who present to Singapore hospitals are seen either at paediatric emergency departments (EDs) staffed by paediatricians and emergency physicians trained in paediatric emergency medicine, or at general EDs staffed by emergency physicians who may have varying training, experience and confidence levels in handling paediatric patients. ${ }^{1,2}$

Paediatric patients presenting to general EDs may differ from those in paediatric EDs in terms of type and acuity of their presenting complaints, thus requiring different treatment procedures and priority of care., Furthermore, the preparedness of general EDs to manage paediatric patients may vary considerably from that of paediatric EDs, thereby affecting the provision of

\footnotetext{
${ }^{1}$ Department of Emergency Medicine, Sengkang General Hospital, Singapore

${ }^{2}$ Accident and Emergency Department, Changi General Hospital, Singapore

${ }^{3}$ Department of Emergency Medicine, KK Women's and Children's Hospital, Singapore

${ }^{4}$ Department of Emergency Medicine, Singapore General Hospital, Singapore

Address for Correspondence: Dr Jen Heng Pek, Department of Emergency Medicine, Sengkang General Hospital, 110 Sengkang East Way, Singapore 544886. Email: pek.jen.heng@singhealth.com.sg
} 
emergency care and clinical outcomes. ${ }^{5-10}$ There is no one-size-fits-all approach to the provision of paediatric emergency care and it will not be realistic for general EDs to have the same capability, function and capacity as paediatric EDs. Therefore, general EDs must first understand the unique characteristics of the paediatric population attending their EDs so they may be better equipped and prepared to deliver appropriate paediatric emergency care based on recommended standards and guidelines. ${ }^{5,11,12}$

In order to address this gap, our study aims at determining the utilisation of EDs by paediatric patients in our healthcare cluster, and elucidating the differences in patterns of utilisation between paediatric and general EDs. In doing so, we will be able to better plan the paediatric emergency services needed within the general EDs, develop training programmes for staff, and establish clinical workflows and processes. Ultimately, the goal would be to enhance the delivery of paediatric emergency care across all EDs in our healthcare cluster.

\section{Methods}

\section{Setting}

In Singapore, the healthcare system is made up of private and public institutions. The public institutions are owned by the government and grouped into 3 healthcare clusters, each covering a specific geographical location. This study was carried out in the largest healthcare cluster consisting of 4 hospitals: a women's and children's paediatric tertiary hospital with a paediatric $\mathrm{ED}$, and 3 adult tertiary hospitals with general EDs. All 4 hospitals are also academic centres. The paediatric $\mathrm{ED}$, staffed by paediatric emergency physicians, is supported by inpatient and outpatient paediatric specialties, whereas the general EDs, staffed by only emergency physicians with different degrees of experience in paediatric emergency care, are not supported by inpatient and outpatient paediatric specialties. Among the 3 general EDs, 2 have the same team of emergency staff attending to paediatric and adult patients in the same space using a single queue system, while 1 has a separate team of emergency staff attending to paediatric patients in a dedicated area using a separate queue system from adult patients. Table 1 shows the characteristics of the 4 hospitals and their EDs.

Pre-hospital emergency medical service for paediatric patients is provided by paramedics from the Singapore Civil Defence Force. The paramedics will assess and provide initial treatment at the incident site before transporting the paediatric patient to 1 of 2 paediatric EDs in Singapore. However, when in extremis due to imminent airway compromise, respiratory failure, profound shock or cardiopulmonary arrest, the paediatric patient will be sent to the nearest ED for stabilisation and treatment before secondary transfer to one of the 2 tertiary paediatric hospitals.

Only the general ED with a dedicated paediatric area has a developed a work process with the paediatric hospital in this study for patients to be directly admitted to its inpatient units. In the other 2 general EDs, paediatric patients who may require inpatient care will be transported to the paediatric hospital for review, before admission to its inpatient units is determined. For these cases, transfers can be made using the caregiver's own transport or regular hospital ambulance. However, if the paediatric patient is critically ill, transfer will be done by the Children's Hospital Emergency Transport Service provided by the paediatric tertiary hospital.

\section{Design}

A retrospective review of all paediatric patients, defined as patients younger than 16 years, who attended the EDs of our healthcare cluster from 1 January 2015 to 31 December 2018 was carried out. Electronic medical records were accessed for data collection using a standardised form. Information including demographics, residential address postal code, mode and time of arrival to the ED, triage category, case type (trauma or nontrauma), disposition, wait time to consultation (time

Table 1. Characteristics of the 4 hospitals and emergency departments

\begin{tabular}{lllll}
\hline Characteristics & Hospital A & Hospital B & Hospital C & Hospital D \\
\hline Type of hospital & Paediatric & Adult & Adult & Adult \\
\hline No. of beds & 830 & 1,000 & 1,000 & 1,700 \\
Type of ED & Paediatric & General & General & General \\
\hline Dedicated paediatric area in ED & Yes & No & Yes & No \\
\hline
\end{tabular}

ED: emergency department

${ }^{a}$ Hospital C began operations in August 2018 
from registration to consultation), length of stay in the ED (time from registration to disposition), as well as mortality outcomes in the EDs were collected.

This study was approved by the Institutional Review Board at SingHealth, Singapore (CIRB reference 2019/2360).

\section{Statistical methods}

Statistical analysis was performed using SPSS Statistics software version 22 (IBM Corp, Armonk, US). Categorical and continuous data were presented as frequencies with percentages and means with standard deviations, respectively. Measures of association were presented using chi-square test for categorical variables and one-way ANOVA for continuous variables. Statistical significance was taken at $P<0.05$.

\section{Results}

\section{Demographics and arrival patterns}

There was a total of $1,893,085$ attendances at the 4 EDs during the study period, of which 704,582 $(37.2 \%)$ were by paediatric patients. The paediatric patients were predominantly seen at the paediatric ED $(686,546,97.4 \%)$ than at the general EDs $(18,036,2.6 \%)$. Overall, a greater number of paediatric patients less than 5 years old were seen at the paediatric ED $(436,097,63.5 \%)$ and general ED with a dedicated paediatric area $(1,166,49.6 \%)$, when compared to general EDs $(2,938,18.7 \%)(P<0.01)$. For patients living outside a $5 \mathrm{~km}$ radius of the hospital, a larger number visited the paediatric ED $(628,621,91.6 \%)$ than the general EDs $(4,482,24.9 \%), P<0.01$. The majority of paediatric attendances at the EDs were self-conveyed $(686,764,97.5 \%)$ and occurred between $4 \mathrm{pm}$ and $12 \mathrm{am}(311,114,44.2 \%)$ (Table 2).

\section{Clinical characteristics and throughput times}

General EDs saw a greater proportion of paediatric patients in the emergent (P1) category $(921,5.1 \%)$ than did paediatric ED $(14,829,2.2 \% ; P<0.01)$. General EDs also saw a greater proportion of paediatric patients with trauma-related presentations $(6,669,37.0 \%)$ than did paediatric ED $(108,822,15.9 \% ; P<0.01)$. The top 5 diagnoses accounting for more than half of all emergent (P1) cases seen at general EDs were seizure

Table 2. Demographics and arrival patterns of paediatric patients at the 4 emergency departments

\begin{tabular}{|c|c|c|c|c|c|}
\hline & Hospital A & Hospital B & Hospital $C^{a}$ & Hospital D & $P$ value \\
\hline Type of ED & Paediatric ED & General ED & $\begin{array}{l}\text { General ED with dedi- } \\
\text { cated paediatric area }\end{array}$ & General ED & - \\
\hline Total no. of attendances & 690,565 & 576,212 & 28,749 & 597,559 & - \\
\hline No. (\%) of paediatric attendances & $686,546(97.4)$ & $11,394(2.0)$ & $2,349(8.2)$ & $4,293(0.7)$ & - \\
\hline \multicolumn{6}{|l|}{ Age range, no. $(\%)$, years } \\
\hline $\begin{array}{l}<1 \\
1 \text { to }<5 \\
5 \text { to }<10 \\
10 \text { to }<16\end{array}$ & $\begin{array}{l}125,845(18.3) \\
310,252(45.2) \\
156,515(22.8) \\
93,934(13.7)\end{array}$ & $\begin{array}{l}71(0.6) \\
1,899(16.7) \\
2,394(21.0) \\
7,030(61.7)\end{array}$ & $\begin{array}{l}198(8.4) \\
968(41.2) \\
639(27.2) \\
544(23.2)\end{array}$ & $\begin{array}{l}289(6.7) \\
679(15.8) \\
753(17.5) \\
2,572(59.9)\end{array}$ & $<0.01$ \\
\hline Stays $>5 \mathrm{~km}$ from ED, no. $(\%)$ & $628,621(91.6)$ & $1,409(12.4)$ & $167(7.1)$ & $2,906(67.7)$ & $<0.01$ \\
\hline $\begin{array}{l}\text { Mode of conveyance, no. (\%) } \\
\text { Own transport } \\
\text { Ambulance }\end{array}$ & $\begin{array}{l}669,227(97.5) \\
17,319(2.5)\end{array}$ & $\begin{array}{l}11,038(96.9) \\
356(3.1)\end{array}$ & $\begin{array}{l}2,326(99.0) \\
23(1.0)\end{array}$ & $\begin{array}{l}4,173(97.2) \\
120(2.8)\end{array}$ & $<0.01$ \\
\hline $\begin{array}{l}\text { Attendance during different time } \\
\text { periods of the day, no. }(\%)^{\mathrm{b}} \\
8 \mathrm{am} \text { to } 4 \mathrm{pm} \\
4 \mathrm{pm} \text { to } 12 \mathrm{am} \\
12 \mathrm{am} \text { to } 8 \mathrm{am}\end{array}$ & $\begin{array}{l}277,756(40.5) \\
301,653(43.9) \\
107,137(15.6)\end{array}$ & $\begin{array}{l}3,414(30.0) \\
6,138(53.9) \\
1,795(15.8)\end{array}$ & $\begin{array}{l}686(29.2) \\
1,236(52.6) \\
427(18.2)\end{array}$ & $\begin{array}{l}1,612(37.5) \\
2,087(48.6) \\
594(13.8)\end{array}$ & $<0.01$ \\
\hline
\end{tabular}

ED: emergency department

${ }^{\text {a }}$ Hospital C began operations in August 2018

${ }^{\mathrm{b}}$ Missing data: Hospital B, 47 (0.4\%) 
(295, 32.0\%), asthma/bronchitis/bronchiolitis ( $83,9.0 \%)$, allergic reaction $(51,5.5 \%)$, cardiac arrest $(48,5.2 \%)$ and burns $(40,4.3 \%)$. While mortality was low across all EDs, general EDs had a higher mortality rate $(39,0.2 \%)$ than paediatric ED $(32,0.005 \% ; P<0.01)$. Trauma-related mortality was uncommon and occurred in $5(12.8 \%)$ and 2 patients $(6.3 \%)$ at the general EDs and paediatric ED, respectively. The mean wait time to consultation of paediatric patients was shorter in paediatric ED $(47.4 \pm 52.1 \mathrm{~min})$ than in general EDs $(51.2 \pm 51.5 \mathrm{~min} ; P<0.01)$. However, the length of stay of paediatric patients was shorter in general EDs $(101.8 \pm 114.8 \mathrm{~min})$ than paediatric EDs $(126.4 \pm 81.3 \mathrm{~min}$; $P<0.01$ ) (Table 3).

\section{Discussion}

The proportion of attendances by paediatric patients varied across the EDs, with paediatric ED receiving the bulk of these patients and general EDs having less than $10 \%$ of paediatric patients in their overall attendances. Despite these lower numbers, the paediatric population at general EDs were of higher acuity with greater mortality rate, and there was a higher proportion of trauma-related presentations. Therefore, paediatric emergency care must remain an integral component of emergency medicine practice, and general EDs must have the necessary capabilities-including manpower and equipment-supported by training and workflow, to attend to the needs of the paediatric population.

The pattern of ED utilisation by paediatric patients in Singapore differed from that in the US where most visits occurred in the general EDs instead of paediatric EDs. The proportion of ED attendance by paediatric patients in paediatric EDs was also higher at $37.2 \%$ in Singapore when compared with $20 \%$ in the US. ${ }^{6}$ These differences were indicative of the care-seeking behaviour of paediatric patients and their family. In an earlier work by Kua et al., up to $60 \%$ of the visits at the paediatric ED were for non-urgent conditions. This observation was attributed to a family's perceived severity of the child's symptoms, availability of after-hours care at the ED, perceived advantage of a paediatric hospital, and reduced confidence of non-paediatricians to manage paediatric conditions. ${ }^{13,14}$ Our study showed similar findings in which the majority of the attendances, particularly those involving patients younger than 5 years, were seen at the paediatric ED rather than at general ED, with almost half of the attendances being low acuity (P3) and presenting during the after-hours for emergency care. Furthermore, by examining the distance between the patient's residential address and ED, we found that the majority of patients who attended paediatric ED lived outside a $5 \mathrm{~km}$ radius of the paediatric ED. This finding was likely unique to Singapore - a small island nation with most locations being within an hour's drive away_making paediatric ED easily accessible and facilitating the caregiver's decision to attend a paediatric ED instead of a general $\mathrm{ED}$, even though the paediatric ED is geographically less convenient.

No 2 EDs are exactly the same, and this should be the case as the capabilities of the ED should be relevant and

Table 3. Clinical characteristics and throughput times of paediatric attendances at the 4 emergency departments

\begin{tabular}{|c|c|c|c|c|c|}
\hline & Hospital A & Hospital B & Hospital Ca & Hospital D & $P$ value \\
\hline Type of ED & Paediatric ED & General ED & $\begin{array}{l}\text { General ED with } \\
\text { dedicated paediatric } \\
\text { area }\end{array}$ & General ED & - \\
\hline Acuity of cases, no. $(\%)^{\mathrm{b}}$ & & & & & $<0.01$ \\
\hline Emergent (P1) & $14,829(2.2)$ & $547(4.8)$ & $44(1.9)$ & $330(7.7)$ & \\
\hline Urgent $(\mathrm{P} 2)$ & $342,141(49.8)$ & $4,321(37.9)$ & $794(33.8)$ & $1,833(42.7)$ & \\
\hline Type of case, no. (\%) & & & & & $<0.01$ \\
\hline Non-trauma & $577,724(84.1)$ & $6,743(59.2)$ & $1,595(67.9)$ & $3,029(70.6)$ & \\
\hline Trauma & $108,822(15.9)$ & $4,651(40.8)$ & $754(32.1)$ & $1,264(29.4)$ & \\
\hline Mortality, no. (\%) & $32(0.005)$ & $24(0.2)$ & $2(0.09)$ & $13(0.3)$ & $<0.01$ \\
\hline Wait time to consult, mean $\pm \mathrm{SD}, \min ^{c}$ & $47.4 \pm 52.1$ & $48.7 \pm 48.4$ & $38.2 \pm 23.6$ & $60.8 \pm 63.5$ & $<0.01$ \\
\hline
\end{tabular}

ED: emergency department; SD: standard deviation

${ }^{\text {a }}$ Hospital C began operations in August 2018

${ }^{\mathrm{b}}$ Missing data: Hospital A, 222 (0.03\%); Hospital B, 14 (0.1\%); Hospital C, 1 (0.04\%); Hospital D, 47 (1.0\%)

${ }^{\mathrm{c}}$ Missing data: Hospital A, 6,628 (1.0\%) 
specific to the needs of the population it serves. When it comes to providing paediatric emergency care, the goal is for general EDs to be prepared and ready to provide appropriate and timely care for paediatric patients arriving through the doors rather than to function with the full capabilities of a paediatric ED. In our study, we have identified 3 key areas of service needs for the general EDs: resuscitation of critically ill paediatric patients, provision of emergency care for trauma-related complaints, and evaluation of common paediatric complaints at the ED.

Our study found that general EDs saw a larger proportion of higher acuity patients than did paediatric EDs. This could be explained by the proximity of EDs as emergency medical service sends critically ill patients to the nearest ED while parents are more likely to take their sick child to the nearest ED. While this larger proportion of higher acuity (P1) patients may have resulted in a correspondingly higher mortality rate in general EDs, it might not be the only reason. Previous studies conducted in the US have shown that critically ill paediatric patients presenting with cardiac or respiratory arrest and major trauma to general EDs had poorer outcomes than those at paediatric EDs. ${ }^{15-18}$ Similarly, the quality of resuscitative care delivered in a simulated setting for paediatric patients with cardiac arrest, sepsis and seizure was also lower for general EDs than for paediatric EDs. ${ }^{19}$ These studies illustrated a variation among EDs in the quality of resuscitative care for paediatric patients with emergent illness and trauma. Therefore, taking into account both the larger proportion of cases and variation in quality of care, paediatric resuscitation should be a key area of focus for general EDs to work on for improving the delivery of paediatric emergency care. General EDs will need to identify and address deficiencies in their processes of care, as well as ensure that staff are trained and material resources are available to handle paediatric resuscitation. At the very least, general EDs should be able to stabilise a critically ill paediatric patient before transfer to a more appropriate facility.

We also found that general EDs treated a larger proportion of trauma-related complaints than did paediatric EDs, a finding similar to an earlier study by Bourgeois and Shannon, which reported that injuries and other musculoskeletal problems made up 35.5\% of paediatric presentations to the general EDs, compared with $20.8 \%$ to paediatric EDs in the US. ${ }^{3}$ This difference could be attributed to parents' belief that injuries can be treated in most EDs; but if their child has a medical complaint such as fever or cough, it would be better for their child to be managed in an institution with paediatric specialists. This overall higher proportion of trauma-related cases seen thus reflects the need for general EDs to be ready and equipped to manage straightforward paediatric trauma cases. This will reduce the number of referrals to the paediatric ED for simple measures such as application of backslab, which can be performed at the general ED. High rates of referrals are associated with increased healthcare costs, additional workload for the receiving ED, and decreased satisfaction of patients, family and providers. ${ }^{20,21}$

The large majority of the paediatric case load, however, remains medical in nature, and thus general EDs would also need to be able to handle common paediatric medical conditions. Sands et al. identified the top presentations to their paediatric ED as breathing difficulty, febrile illness, diarrhoea with or without vomiting, rash and cough. ${ }^{22}$ While this may serve as a general guide for planning, each ED needs to analyse its pattern of paediatric presentations to know how best to prepare its department. ${ }^{23}$ This approach would allow general EDs to build on their capabilities in a progressive manner, beginning with development of problem-based guidelines supported by best evidence to improve quality of clinical care and training programme to ensure that a critical mass of their staff would be able to handle the most commonly encountered paediatric complaints before progressing to gaining proficiency in less common conditions. ${ }^{24}$

Finally, we also identified that the mean wait time to consultation was shorter in paediatric ED than in the general EDs. However, the length of stay was longer in paediatric ED than in the general EDs. Time to consult and length of stay are important quality and patient satisfaction indicators for EDs, which have been identified in various studies. ${ }^{25,26} \mathrm{~A}$ long wait time may lead to patients leaving prior to consult, which may in turn jeopardise the provision of timely care to patients who need it the most. ${ }^{27,28}$ Therefore, EDs should try to minimise the wait time for patients whenever possible. We postulated that these differences could be due to unique queue systems in place and management objectives of the EDs. For instance, the general ED with a dedicated paediatric area had the shortest wait time to consultation among the general EDs. This observation was likely attributable to having a separate team to attend to only paediatric patients, which allowed them to receive consultation for their conditions faster, suggesting a benefit over the queue system in other EDs, which kept paediatric patients together with adult patients with differing needs at the ED, thus resulting in a longer time to consult for paediatric patients. For length of stay in 
the $\mathrm{ED}$, it was likely related to management objectives of the clinicians at the EDs, and these could range from a complete and thorough evaluation with treatment of underlying conditions at paediatric ED, to a focused assessment and stabilisation if required at general EDs with onward referral to a paediatric ED for further care.

\section{Limitations}

This study was carried out in a single healthcare cluster. Even though our cluster includes Singapore's largest paediatric and adult hospitals with the highest ED attendances, a more in-depth understanding of the utilisation of EDs by paediatric patients would warrant a nationwide collaborative effort involving multiple institutions and other healthcare clusters. Also, our healthcare cluster consisted only of tertiary hospitals and academic centres in urban areas. As such, other hospitals such as community and non-academic centres were not represented, and we were unable to share insight into how the use of EDs in these centres by the paediatric population would vary.

Next, this was a retrospective study based on the review of patients' electronic medical records documented by various medical personnel. This means that there would be inconsistency in documentation, leading to missing or incomplete information that we were unable to verify. Furthermore, we were unable to include all outcomes - such as ED re-attendance and morbiditiesdue to occurrence of adverse events like misdiagnoses and medication errors that may be relevant to this study, as we had to work within the restrictions of a retrospective dataset. Looking forward, a national prospective study involving all EDs across healthcare clusters, as well as public and private institutions, will be useful to seek further clarity on the issues identified.

In conclusion, this study has provided us with a better understanding of the utilisation of EDs by paediatric patients and has demonstrated the difference in patterns of use between general and paediatric EDs. This knowledge is actionable and may translate to better emergency care for paediatric patients as EDs take steps towards building their capability and enhancing their preparedness.

\section{REFERENCES}

1. Green SM, Ruben J. Emergency department children are not as sick as adults: implications for critical care skills retention in an exclusively pediatric emergency medicine practice. J Emerg Med 2009;37:359-68.

2. Langhan M, Keshavarz R, Richardson LD. How comfortable are emergency physicians with pediatric patients? J Emerg Med 2004;26:465-9.
3. Bourgeois FT, Shannon MW. Emergency care for children in pediatric and general emergency departments. Pediatr Emerg Care 2007;23:94-102.

4. Hudgins JD, Monuteaux MC, Bourgeois FT, et al. Complexity and severity of pediatric patients treated at United States emergency departments. J Pediatr 2017; 186:145-9.e1.

5. Remick K, Gausche-Hill M, Joseph MM, et al. Pediatric readiness in the emergency department. Ann Emerg Med 2018;72:e123-36.

6. Ames SG, Davis BS, Marin JR, et al. Emergency department pediatric readiness and mortality in critically ill children. Pediatrics 2019; 144:e20190568.

7. Remick K, Gaines B, Ely M, et al. Pediatric emergency department readiness among US trauma hospitals. J Trauma Acute Care Surg 2019;86:803-9.

8. Remick K, Kaji AH, Olson L, et al. Pediatric readiness and facility verification. Ann Emerg Med 2016;67:320-8.e1.

9. Gausche-Hill M, Ely M, Schmuhl P, et al. A national assessment of pediatric readiness of emergency departments. JAMA Pediatr 2015;169:527-34.

10. Jain PN, Choi J, Katyal C. Pediatric care in the non-pediatric emergency department: provider perspectives. Hosp Pediatr 2019;9:216-9.

11. Royal College of Paediatrics and Child Health. Facing the future: standards for children in emergency care settings, June 2018. Available at: https://www.rcpch.ac.uk/resources/facing-futurestandards-paediatric-care. Accessed on 12 September 2020.

12. International Federation for Emergency Medicine. 2012 international standards of care for children in emergency departments, updated June 2014. Available at: https://www.ifem.cc/wp-content/ uploads/2016/03/International-Standards-for-Children-inEmergency-Departments-V2.0-June-2014-1.pdf. Accessed on 12 September 2020.

13. Kua PH, Wu L, Ong EL, et al. Understanding decisions leading to nonurgent visits to the pediatric emergency department: caregivers' perspectives. Singapore Med J 2016;57:314-9.

14. Ganapathy S, Lim SY, Kua JP, et al. Non-urgent paediatric emergency department visits: why are they so common? A Singapore perspective. Ann Acad Med Singap 2015;44:269-71.

15. Athey J, Dean JM, Ball J, et al. Ability of hospitals to care for pediatric emergency patients. Pediatr Emerg Care 2001;17:170-4.

16. Hansen M, Fleischman R, Meckler G, et al. The association between hospital type and mortality among critically ill children in US EDs. Resuscitation 2013;84:488-91.

17. Sathya C, Alali AS, Wales PW, et al. Mortality among injured children treated at different trauma center types. JAMA Surg 2015;150:874-81

18. Michelson KA, Hudgins JD, Monuteaux MC, et al. Cardiac arrest survival in pediatric and general emergency departments. Pediatrics 2018;141:e20172741.

19. Auerbach M, Whitfill T, Gawel M, et al. Differences in the quality of pediatric resuscitative care across a spectrum of emergency departments. JAMA Pediatr 2016;170:987-94.

20. Hoa MHL, Ong YG, Pek JH. Trauma transfers to the pediatric emergency department - is it necessary? Turk J Emerg Med 2020;20:12-7.

21. Newgard CD, Staudenmayer K, Hsia RY, et al. The cost of overtriage: more than one-third of low-risk injured patients were taken to major trauma centers. Health Aff (Millwood) 2013; $32: 1591-9$. 
22. Sands R, Shanmugavadivel D, Stephenson T, et al. Medical problems presenting to paediatric emergency departments: 10 years on. Emerg Med J 2012;29:379-82.

23. Armon K, MacFaul R, Hemingway P, et al. The impact of presenting problem based guidelines for children with medical problems in an accident and emergency department. Arch Dis Child 2004;89:159-64.

24. Armon K, Stephenson T, Gabriel V, et al. Determining the common medical presenting problems to an accident and emergency department. Arch Dis Child 2001;84:390-2.
25. Magaret ND, Clark TA, Warden CR, et al. Patient satisfaction in the emergency department - a survey of pediatric patients and their parents. Acad Emerg Med 2002;9:1379-88.

26. Bursch B, Beezy J, Shaw R. Emergency department satisfaction: what matters most? Ann Emerg Med 1993;22:586-91.

27. Bindman AB, Grumbach K, Keane D, et al. Consequences of queuing for care at a public hospital emergency department. JAMA 1991;266:1091-6.

28. Baker DW, Stevens CD, Brook RH. Patients who leave a public hospital emergency department without being seen by a physician: causes and consequences. JAMA 1991;266:1085-90. 